SERIE B - INFORMATIK

\title{
A Ramsey-type theorem in the plane
}

\author{
Jaroslav Nešetřil ${ }^{*}$ \\ Pavel Valtr* ${ }^{\dagger}$ \\ B 94-03 \\ January 1994
}

\begin{abstract}
We show that for any finite set $P$ of points in the plane and for any integer $k \geq 2$ there is a finite set $R=R(P, k)$ with the following property: For any $k$-colouring of $R$ there is a monochromatic set $\tilde{P}, \tilde{P} \subseteq R$, such that $\tilde{P}$ is combinatorially equivalent to the set $P$ and the convex hull of $\tilde{P}$ contains no point of $R \backslash \tilde{P}$. We also consider related questions for colourings of $p$-element subsets of $R(p>1)$ and show that these analogues have negative solutions.
\end{abstract}

*Department of Applied Mathematics, Charles University, Malostranské nám. 25, 11800 Praha 1, Czech republic.

†Graduiertenkolleg "Algorithmische Diskrete Mathematik", Fachbereich Mathematik, Freie Universität Berlin, Takustrasse 9, 14195 Berlin, Germany, supported by "Deutsche Forschungsgemeinschaft", grant We $1265 / 2-1$. 


\section{Introduction and the statement of results}

In this paper we investigate geometrical Ramsey-type results which are related to the celebrated Erdös-Szekeres Theorem.

Theorem 1 (Erdös-Szekeres Theorem) For every positive integer $n$ there exists a positive integer ES( $n)$ such that any set $X$ of $E S(n)$ points in general position in the plane (i.e., no three lie on a line) contains vertices of a convex n-gon.

The Erdös-Szekeres Theorem is one of the original gems of Ramsey theory. By combining it with Ramsey's theorem [Ra 30] (see also [GRS 80] or [NR 90]) itself we get:

Corollary 2 For every choice of positive integers $p, k, n$, there exists a positive integer $\operatorname{ES}(p, k, n)$ with the following property: For any set $X$ of at least $\operatorname{ES}(p, k, n)$ points in general position in the plane and for any partition $\left(\begin{array}{l}X \\ p\end{array}\right)=C_{1} \cup \ldots \cup C_{k}$ there exists a set $Y \subseteq X,|Y|=n$, such that all p-subsets of $Y$ belong to one class $C_{i_{0}}$ of the partition and $Y$ is the set of vertices of a convex $n$-gon.

To verify the corollary, one simply puts $E S(p, k, n)=r(p, k, E S(n))$, where $r(p, k, N)$ is the usual Ramsey number for $p$-tuples and $k$ colours.

We are interested in a generalization of Corollary 2 to sets $Y$ of a given configuration. Somewhat surprisingly this generalization can be done for $p=1$ (i.e., for partition of points) while in general $(p>1)$ a similar statement fails to be true.

Here is the key concept of this paper: Two finite planar point sets $P$ and $Q$ are called combinatorially equivalent if there exists a bijection $i: P \rightarrow Q$ such that $p \in \operatorname{conv}\left(P^{\prime}\right)$ if and only if $i(p) \in \operatorname{conv}\left(i\left(P^{\prime}\right)\right)$, for any $p \in P$ and $P^{\prime} \subseteq P$. Here $\operatorname{conv}(X)$ is the convex hull of the set $X$ and $i(X)$ denotes the set $\{i(x): x \in X\}$.

A finite planar point set $X$ is said to be convex independent if $\operatorname{conv}\left(X^{\prime}\right) \neq$ $\operatorname{conv}(X)$ for every proper subset $X^{\prime}$ of $X$ (or equivalently, if points of $X$ are vertices of a convex polygon). Otherwise $X$ is said to be convex dependent. It is easy to see that two sets are combinatorially equivalent if and only if there is a bijection between them which preserves both convex dependent and convex independent sets.

Combinatorially equivalent sets and similar concepts have already been studied explicitly in several papers (see [GP 91] or [GP 93] for a survey). Implicitly they play a very important role mainly in discrete and computational geometry. See also [KLS 91] for a survey on more general structures. In Section 2 we prove the following Ramsey-type theorem.

Theorem 3 For any finite set $P$ of points in the plane and for any integer $k \geq 2$, there is a finite set $R=R(P, k)$ of points in the plane such that for any partition of $R$ into $k$ colour classes there is a subset $\tilde{P}$ of $R$ with the following three properties:

(i) $\tilde{P}$ is monochromatic (i.e., it is a subset of one of the colour classes),

(ii) $\tilde{P}$ is combinatorially equivalent to $P$,

(iii) $\operatorname{conv}(\tilde{P}) \cap R=\tilde{P}$. 
Moreover, as we shall see from the proof, the set $\tilde{P}$ in Theorem 3 may be required to be an affine transform of $P$.

A subset $X$ of a finite planar point set $P$ is called a hole in $P$, or simply a $P$-hole, or an empty polygon, if $X$ is convex independent and $\operatorname{conv}(X) \cap P=X$. Horton [Ho 83] (see also [BF 87] or [Va 92]) proved that, for $n \geq 7$, the ErdösSzekeres Theorem cannot be strengthened to guarantee the existence of an $n$-hole. The existence of a 5-hole in any set of 10 points in general position in the plane was shown by Harborth [Ha 78] while the case $n=6$ is still open.

Now Theorem 3 can be rephrased as follows.

Theorem 4 For any finite set $P$ of points in the plane and for any integer $k \geq 2$, there is a finite set $R=R(P, k)$ of points in the plane such that for any partition $R=C_{1} \cup \ldots \cup C_{k}$ there is an injection $i: P \rightarrow R$ with the following three properties: (i) $i(P) \subseteq C_{i_{0}}$ for some $i_{0} \in\{1, \ldots, k\}$,

(ii) $i$ preserves convex independent and convex dependent sets,

(iii) i maps P-holes to R-holes.

Motivated by Corollary 2 one can also consider a higher-order Ramsey-type theorem (for $p>1$ ). If we are partitioning the set $\left(\begin{array}{l}R \\ 2\end{array}\right)$ into $k$ colour classes then a result analogous to Theorem 3 cannot be valid in general: The pairs of any finite set $R$ can be coloured as follows. On every line with at least two points of $R$ we colour pairs of consecutive points alternately by two colours while all the other pairs are coloured arbitrarily. In this way we avoid a monochromatic triple of collinear points of $R$ containing no other point of $R$ in the convex hull. It follows that if we are partitioning the set $\left(\begin{array}{c}R \\ 2\end{array}\right)$ then a result analogous to Theorem 3 cannot be valid for planar point sets $P$ which are not in general position. More generally, for $p \geq 2$, if we are partitioning the set $\left(\begin{array}{l}R \\ p\end{array}\right)$ then a result analogous to Theorem 3 cannot be valid for planar point sets $P$ with $p+1$ points on a line.

However, the situation is more difficult if we restrict our attention to point sets $P$ in general position. It turns out that Theorem 3 has no higher-order analogues even in this case. In fact, this remains true even if we drop the hole-preserving condition:

Theorem 5 For every $p \geq 2$, there exists a finite planar point set $P(p)$ in general position with the following property: There exists a partition $\left(\begin{array}{c}\mathbb{R}^{2} \\ p\end{array}\right)=C_{1} \cup C_{2}$ of all p-element subsets of the plane into two colour classes such that no monochromatic subset of $\mathbb{R}^{2}$ is combinatorially equivalent to $P(p)$.

Thus Theorem 3 fails to have a higher-order analogue in general. However, such an analogue holds in some particular cases. Apart from Corollary 2 (which yields such an analogue for any finite convex independent set $P$ ) we have the following result which deals with the configuration $Q$ containing the three vertices and an inner point of a triangle. 
Theorem 6 Let $Q$ be a convex dependent set of four points in general position in the plane. Then, for every integer $k \geq 1$, there exists a finite planar point set $R=R(k)$ such that for every partition of pairs of $R$ into $k$ classes one of the classes contains all 6 pairs of points of some 4-point set combinatorially equivalent to $Q$.

The paper is divided into sections as follows. Section 2 contains the proof of Theorem 3. In Section 3 we give the proofs of Theorems 5 and 6 . Section 4 contains concluding remarks.

\section{Proof of the main result}

Here is a short outline of the proof of Theorem 3: Given a planar point set we find an equivalent set $\tilde{P}$ whose points are placed inside a small neighborhood of a line. Then we construct a set $R$ containing many subsets combinatorially equivalent to $\tilde{P}$ and apply the Hales-Jewett Theorem to show that at least one of these subsets is monochromatic.

Fix a set $P$ in the plane, and let $n=|P|$. Without loss of generality (or by a slight rotation of $P$ ) we may assume that all $x$-coordinates of the set $P$ are different. Let the points of $P$, ordered according to their $x$-coordinate, be $p(1), \ldots, p(n)$.

Let $M>0$ be any number such that all points of the set $P$ lie inside the $M$ neighborhood of the $x$-axis (i.e., the $y$-coordinates of the points in $P$ lie in the interval $(-M, M))$. For any $\varepsilon \geq 0$, let $P_{\varepsilon}$ be the set obtained from the set $P$ by replacing each point $(x, y)$ by the point $\left(x, \frac{\varepsilon}{M} y\right)$. If $\varepsilon \neq 0$ then the set $P_{\varepsilon}$ is equivalent to $P$ and is placed inside the $\varepsilon$-neighborhood of the $x$-axis. Otherwise (if $\varepsilon=0$ ) the points of $P_{0}$ are collinear. The points of $P_{\varepsilon}$ listed according to increasing $x$-coordinate will be denoted by $p_{\varepsilon}(1), \ldots, p_{\varepsilon}(n)$.

Let $\alpha \in[0, \pi)$ and let $r_{\alpha}$ be the rotation of the plane by the angle $\alpha$ around the origin. Thus $r_{\alpha}(x)$ denotes the point which we get by rotating $x$ by $r_{\alpha}$. Put $p_{\varepsilon, \alpha}(i)=r_{\alpha}\left(p_{\varepsilon}(i)\right)$ (for $\left.\varepsilon \geq 0, i=1, \ldots, n\right)$ and $P_{\varepsilon, \alpha}=\left\{p_{\varepsilon, \alpha}(1), \ldots, p_{\varepsilon, \alpha}(n)\right\}$ (for $\varepsilon \geq 0)$. Thus we could also write $P_{\varepsilon, \alpha}=r_{\alpha}\left(P_{\varepsilon}\right)$.

To invoke the Hales-Jewett Theorem we need some notation. Put $A=\{1, \ldots, n\}$ (and think of $A$ as an alphabet). Given a positive integer $N$, we consider the set $A^{N}$ of all mappings $\{1, \ldots, N\} \rightarrow A$. One can also think of $A^{N}$ as of the $N$-dimensional cube over $A$. A (combinatorial) line $L$ in $A^{N}$ is defined as an $n$-element subset of $A^{N}$ satisfying the following condition: There exists a proper subset $\omega \subset\{1, \ldots, N\}$ and a mapping $f_{0}: \omega \rightarrow A$ such that the line $L$ is formed by all mappings $f$ : $\{1, \ldots, N\} \rightarrow A$ which satisfy $f(i)=f_{0}(i)$ for $i \in \omega$ and $f(i)=f(j)$ whenever $i, j \notin \omega$. More explicitly, $L=\{x(1), \ldots, x(n)\}$, where (for $j=1, \ldots, n$ )

$$
x(j)=\left(x_{1}(j), \ldots, x_{N}(j)\right)
$$

and

$$
x_{i}(j)= \begin{cases}f_{0}(i) & \text { for } i \in \omega \\ j & \text { for } i \notin \omega .\end{cases}
$$


Now we can formulate the Hales-Jewett Theorem [HJ 63] (see also [GRS 80] or [NR 90]).

Theorem 7 (Hales-Jewett Theorem) For any two positive integers $n, k \geq 2$, there exists an integer $N=N(n, k)$ such that for any $k$-colouring of the points of the cube $A^{N}, A=\{1, \ldots, n\}$, there exists a monochromatic line.

Denote by $\mathcal{L}\left(A^{N}\right)$ the set of all (combinatorial) lines in $A^{N}$. For two points $x=\left(x_{1}, x_{2}\right), y=\left(y_{1}, y_{2}\right)$ in the plane, we define $x+y=\left(x_{1}+y_{1}, x_{2}+y_{2}\right)$.

We shall embed the cube $A^{N}$ into the plane using properly chosen rotations determined by 'independent' angles. For simplicity, for $\varepsilon \geq 0, \alpha=\left(\alpha_{i}\right)_{1}^{N}$, and $x=$ $\left(x_{i}\right)_{1}^{N} \in A^{N}$, put $q_{\varepsilon, \alpha}(x)=\sum_{i=1}^{N} p_{\varepsilon, \alpha_{i}}\left(x_{i}\right)$ and $q_{\alpha}(x)=q_{0, \alpha}(x)$. Now let $L \in \mathcal{L}\left(A^{N}\right)$ be a combinatorial line such that the points $q_{\alpha}(x)(x \in L)$ are distinct. It is easy to check that the points $q_{\alpha}(x)(x \in L)$ all lie on a straight line on the plane. Let us denote this line by $q_{\alpha}(L)$. We say that $\alpha=\left(\alpha_{1}, \ldots, \alpha_{N}\right)$ above is $P$-independent if the points $q_{\alpha}(x)\left(x \in A^{N}\right)$ are distinct and $q_{\alpha}(x) \notin q_{\alpha}(L)$ for all lines $L \in \mathcal{L}\left(A^{N}\right)$ and all $x \in A^{N} \backslash L$.

Lemma 8 For any finite planar point set $P$ with different $x$-coordinates and for any positive integer $N$, there is a P-independent $N$-tuple $\alpha=\left(\alpha_{1}, \ldots, \alpha_{N}\right)$.

Proof. Let $\alpha=\left(\alpha_{1}, \ldots, \alpha_{N}\right)$ be an arbitrary tuple of $N$ angles. We shall show that one can get a $P$-independent $N$-tuple by small change of the angles $\alpha_{i}$. Suppose two points $q_{\alpha}\left(x_{1}\right), q_{\alpha}\left(x_{2}\right)\left(x_{1}, x_{2} \in A^{N}, x_{1} \neq x_{2}\right)$ coincide. Let $x_{1}$ and $x_{2}$ differ in the $i$-th coordinate. For $\varepsilon>0$, set $\bar{\alpha}=\left(\alpha_{1}, \ldots, \alpha_{i-1}, \alpha_{i}+\varepsilon, \alpha_{i+1}, \ldots, \alpha_{N}\right)$. The points $q_{\bar{\alpha}}\left(x_{1}\right)$ and $q_{\bar{\alpha}}\left(x_{2}\right)$ are different and, if $\varepsilon>0$ is sufficiently small, any two points $q_{\bar{\alpha}}(x), q_{\bar{\alpha}}\left(x^{\prime}\right), x, x^{\prime} \in A^{N}$, are different whenever the points $q_{\alpha}(x)$ and $q_{\alpha}\left(x^{\prime}\right)$ are different. Thus, if $\alpha$ is replaced by $\bar{\alpha}$ (with $\varepsilon>0$ sufficiently small) then the number of coincidences between the points $q_{\alpha}(x)\left(x \in A^{N}\right)$ drops. Repeating this procedure we obtain a tuple $\alpha$ such that the points $q_{\alpha}(x)\left(x \in A^{N}\right)$ are distinct.

Suppose now that, for $L_{0} \in \mathcal{L}\left(A^{N}\right)$ and for $x_{0} \in A^{N} \backslash L_{0}$, the point $q_{\alpha}\left(x_{0}\right)$ lies on the line $q_{\alpha}\left(L_{0}\right)$. Let $i \in\{1, \ldots, N\}$ be such that the $n$ points of $L_{0}$ have distinct $i$-th coordinates (i.e., their $i$-th coordinates are 1 through $n$ ). For $\varepsilon>0$, set $\bar{\alpha}=\left(\alpha_{1}, \ldots, \alpha_{i-1}, \alpha_{i}+\varepsilon, \alpha_{i+1}, \ldots, \alpha_{N}\right)$ as above.

Suppose $q_{\alpha}\left(L_{0}\right)$ determines the angle $\beta$ with the positive $x$-axis. Picking small enough $\varepsilon>0$, we may assume that $\left(\alpha_{i}+\left(\alpha_{i}+\varepsilon\right)\right) / 2 \neq \beta-\pi / 2$ and $\left(\alpha_{i}+\left(\alpha_{i}+\varepsilon\right)\right) / 2 \neq$ $\beta+\pi / 2$. Then, $q_{\alpha}\left(L_{0}\right)$ and $q_{\bar{\alpha}}\left(L_{0}\right)$ are not parallel. Let $\hat{x}$ be the point of $L_{0}$ whose $i$-th coordinate equals the $i$-th coordinate of $x_{0}$. Then, the points $q_{\bar{\alpha}}(\hat{x})$ and $q_{\bar{\alpha}}\left(x_{0}\right)$ lie on a line which is parallel to $q_{\alpha}\left(L_{0}\right)$ and, hence, not parallel to $q_{\bar{\alpha}}\left(L_{0}\right)$. Note that the point $q_{\bar{\alpha}}(\hat{x})$ lies on the line $q_{\bar{\alpha}}\left(L_{0}\right)$ by definition. It follows that the point $q_{\bar{\alpha}}\left(x_{0}\right)$ does not lie on the line $q_{\bar{\alpha}}\left(L_{0}\right)$ (since otherwise $q_{\bar{\alpha}}\left(x_{0}\right)=q_{\bar{\alpha}}(\hat{x})$ and, consequently, $\left.q_{\alpha}\left(x_{0}\right)=q_{\alpha}(\hat{x})\right)$. If $\varepsilon>0$ is sufficiently small then the points $q_{\bar{\alpha}}(x)\left(x \in A^{N}\right)$ are distinct and, for any $L \in \mathcal{L}\left(A^{N}\right)$ and $x \in A^{N} \backslash L$, the point $q_{\bar{\alpha}}(x)$ does not lie on the line $q_{\bar{\alpha}}(L)$ whenever the point $q_{\alpha}(x)$ does not lie on the line $q_{\alpha}(L)$. Thus, if 
$\alpha$ is replaced by $\bar{\alpha}$ (with $\varepsilon>0$ sufficiently small) then the number of line-point incidences drops.

Repeating the procedure described in the above two paragraphs, we finally obtain a $P$-independent tuple $\alpha$.

Now we are in a position to conclude the proof of Theorem 3.

Proof of Theorem 3. Let $P$ be a set of $n$ points in the plane with distinct $x$ coordinates. Put $A=\{1, \ldots, n\}$. Let $N=N(n, k)$ be a number guaranteed by the Hales-Jewett Theorem. Let $\alpha$ be a $P$-independent $N$-tuple. Thus for any line $L \in \mathcal{L}\left(A^{N}\right)$ and any $x \in A^{N} \backslash L$ we have $q_{\alpha}(x) \notin q_{\alpha}(L)$. By an obvious continuity argument, there is a sufficiently small $\varepsilon>0$ such that, for any line $L \in \mathcal{L}\left(A^{N}\right)$, the convex hull of the points $q_{\varepsilon, \alpha}(x)(x \in L)$ contains no point $q_{\varepsilon, \alpha}(x)$ with $x \in A^{N} \backslash L$. For any line $L \in \mathcal{L}\left(A^{N}\right)$, the set $\left\{q_{\varepsilon, \alpha}(x): x \in L\right\}$ is combinatorially equivalent to the set $P$. Now we are prepared to show that the set $R=\left\{q_{\varepsilon, \alpha}(x): x \in A^{N}\right\}$ has all the required properties.

Let $R=C_{1} \cup \ldots \cup C_{k}$ be a $k$-colouring of $R$. It induces a $k$-colouring of the set $A^{N}$. According to the Hales-Jewett Theorem, there exists a monochromatic line $L_{0} \in \mathcal{L}\left(A^{N}\right)$. The set $\tilde{P}=q_{\varepsilon, \alpha}\left(L_{0}\right)=\left\{q_{\varepsilon, \alpha}(x): x \in L_{0}\right\}$ is a monochromatic subset of $R$, it is combinatorially equivalent to $P$, and its convex hull contains no point of $R \backslash \tilde{P}$.

Note that the set $\tilde{P}$ in the above proof is actually an affine transform of the set $P$.

\section{Proofs of related results}

In this section we prove Theorems 5 and 6 . The proof of Theorem 5 follows from the following geometric result which we proved in [NV 93].

Theorem 9 ([NV 93]) For any integer $k>0$ and for any $k+1$ positive real numbers $\varepsilon, r_{1}, r_{2}, \ldots, r_{k}>0$, there exists a finite planar point set $P$ in general position such that any set combinatorially equivalent to $P$ determines $k+1$ distances $d_{i}(i=$ $0,1, \ldots, k)$ such that $\left|r_{i}-d_{i} / d_{0}\right|<\varepsilon$ for any $i=1,2, \ldots, k$. Moreover, one may require the distances $d_{i}(i=0,1, \ldots, k)$ to be determined by pairwise disjoint pairs of points.

Proof of Theorem 5. First we prove Theorem 5 for $p=2$. Let $P$ be a set satisfying Theorem 9 for $k=2, \varepsilon=0.01, r_{1}=1.9$, and $r_{2}=2.5$. We find a 2 -colouring of all pairs of points of the plane such that no set combinatorially equivalent to $P$ has all pairs coloured by the same colour. Any pair $(x, y) \in\left(\begin{array}{c}\mathbb{R}^{2} \\ 2\end{array}\right)$ of points of the plane with Euclidean distance $|x y|>0$ will be coloured blue if $\left.\left|\log _{2}\right| x y \mid\right\rfloor$ is an even integer. Otherwise $(x, y)$ will be coloured red. In other words, a pair of points is coloured 
blue if and only if the distance between the two points belongs to some interval $\left[2^{t}, 2^{t+1}\right)$, where $t$ is an even integer.

Now let $P^{\prime}$ be a set combinatorially equivalent to $P$ and let $d_{i}, i=0,1,2$, be the three distances in $P^{\prime}$ ensured by Theorem 9 . Thus $\left|\frac{d_{1}}{d_{0}}-1.9\right|<0.01$. If the two pairs of points determining $d_{0}$ and $d_{1}$ are coloured by the same colour, then the numbers $d_{0}$ and $d_{1}$ belong to the same interval $\left[2^{t}, 2^{t+1}\right), t \in \mathbb{Z}$, and, consequently, $d_{2}$ belongs to the next interval $\left[2^{t+1}, 2^{t+2}\right)$. It follows that all the three pairs determining the distances $d_{0}, d_{1}, d_{2}$ cannot be coloured by the same colour. Theorem 5 for $p=2$ follows.

Now let $p>2$. Fix an arbitrary linear order $\prec$ of the points of the plane, and colour every $p$-tuple of points of the plane by the colour in which the pair of the two smallest (in the order $\prec$ ) points of the $p$-tuple was coloured above. A short argument shows that Theorem 5 holds for this 2-colouring and for the set $P(p)=P$ obtained from Theorem 9 for $k=3 p-4, \varepsilon=0.01, r_{1}=\ldots=r_{p-2}=1$, $r_{p-1}=\ldots=r_{2 p-3}=1.9$, and $r_{2 p-2}=\ldots=r_{3 p-4}=2.5$.

Thus, an analogue of Theorem 3 fails to be true for partitions of $p$-tuples in a very strong sense. However, some particular cases are valid. One such example is provided by Theorem 6 . We could denote the statement of Theorem 6 by $R \rightarrow(Q)_{k}^{2}$. Despite the simplicity of the configuration $Q$ our proof is quite involved and, in particular, it makes use of Theorem 3. We shall only sketch the proof here as we intend to return to this topic elsewhere.

Proof of Theorem 6 (Sketch).

First we prove the following lemma.

Lemma 10 For every given point sets $P_{1}, P_{2}$ in general position and for every $k \geq 1$, there are point sets $R_{1}, R_{2}$ with the following two properties:

(i) $R_{1} \cup R_{2}$ is in general position,

(ii) for every partition $C_{1} \cup \ldots \cup C_{k}$ of all pairs $\left(x_{1}, x_{2}\right), x_{1} \in R_{1}, x_{2} \in R_{2}$, there exist two sets $P_{1}^{\prime} \subseteq R_{1}, P_{2}^{\prime} \subseteq R_{2}, P_{i}^{\prime}$ combinatorially equivalent to $P_{i}$ for $i=1,2$, such that all pairs $\left(x_{1}, x_{2}\right), x_{1} \in R_{1}, x_{2} \in R_{2}$, are monochromatic.

Proof. We apply Theorem 3. Put $R_{1} \rightarrow\left(P_{1}\right)_{k}^{1}, R_{2} \rightarrow\left(P_{2}\right)_{K}^{1}$, where $K=k^{\left|R_{1}\right|}$. By a standard Ramsey theory argument we get the statement.

Clearly Lemma 10 may be generalized (from bipartite to multipartite graphs with more sets $\left.P_{1}, P_{2}, P_{3}, \ldots\right)$. We shall use this for $r$-partite graphs, where $r=r_{k}(3)$ is the classical Ramsey number for a monochromatic triangle in any $k$-colouring of the edges of the complete graph.

Somewhat surprisingly we shall prove Theorem 6 by induction on $k$ : For $k=1$ Theorem 6 trivially holds. Let us assume that we have already found a planar point set $S$ such that $S \rightarrow(Q)_{k-1}^{2}$. Now let $R_{1}, \ldots, R_{r}$ be $r$ planar point sets such that for any partition of all pairs $(x, y), x \in R_{i}, y \in R_{j}, i \neq j$, there are $r$ sets 
$S_{1}, \ldots, S_{r}, S_{1} \subseteq R_{1}, \ldots, S_{r} \subseteq R_{r}$, equivalent to $S$ such that for every choice of indices $i, j, i \neq j$, all pairs between $S_{i}$ and $S_{j}$ are coloured by the same colour $c(i, j)$. Now we can suppose that the set $R_{i}$ is placed in a very small neighborhood of the vertical line $L_{i}=\{(i, y): y \in \mathbb{R}\}$ with all its $y$-coordinates distinct. Assume that the $y$-coordinates of all the points in $R_{i}$ are in the interval $\left(i^{2}, i^{2}+1\right)$. According to Ramsey's Theorem $\left(r=r_{k}(3)\right)$, there are three indices $i, j, l, i<j<l$, such that all pairs $x, y$ between $S_{i}, S_{j}, S_{l}$ are coloured by the same colour $c$ (in the above notation $c=c(i, j)=c(i, l)=c(j, l))$. If no pair of distinct points of $S_{j}$ is coloured by the colour $c$, then we can use the inductive assumption $S_{j} \rightarrow(Q)_{k-1}^{2}$ to get a copy of $Q$ with all pairs of points coloured by the same colour. Thus we may assume that there exists a pair $\left(x_{j}, x_{j}^{\prime}\right)$ of points of $S_{j}$ coloured by the colour $c$. Choose $x_{i} \in S_{i}, x_{l} \in S_{l}$ arbitrarily. By our construction both $x_{j}$ and $x_{j}^{\prime}$ lie below the line $x_{i} x_{l}$ and, if $R_{i}$ is in a small enough neighborhood of $L_{i}$, the line $x_{j} x_{j}^{\prime}$ separates the points $x_{i}$ and $x_{l}$. Thus $\left\{x_{i}, x_{j}, x_{j}^{\prime}, x_{l}\right\}$ is a homogeneous set equivalent to $Q$.

\section{Concluding remarks}

1. Theorem 3 may be generalized to any fixed finite dimension. Since Theorem 9 holds in a higher dimension (see [NV 93]), Theorem 5 may be also generalized to a higher dimension.

2. Another way of rephrasing Theorem 3 (for sets in general position) is by means of geometric graphs (which were studied in various contexts, e.g. in [Lo 79], [NPT 81], [KL 85], [Pa 91], [MP 93]). A geometric graph is defined as a pair ( $V, E)$, where $V$ is a set of points in general position in the plane and $E$ is a subset of the set of all line segments connecting points of $V$. Two geometric graphs $(V, E),\left(V^{\prime}, E^{\prime}\right)$ are said to be isomorphic if there exists a bijection $f: V \rightarrow V^{\prime}$ satisfying the following two conditions:

(i) $v_{1} v_{2} \in E$ if and only if $f\left(v_{1}\right) f\left(v_{2}\right) \in E^{\prime}$,

(ii) two line segments $v_{1} v_{2}, v_{3} v_{4} \in E$ cross if and only if the corresponding line segments $f\left(v_{1}\right) f\left(v_{2}\right), f\left(v_{3}\right) f\left(v_{4}\right) \in E^{\prime}$ cross.

The following theorem may be proved with the method used in the proof of Theorem 3.

Theorem 11 For every geometric graph $(V, E)$ there exists a geometric graph $(W, F)$ such that, for every partition $W=C_{1} \cup \ldots \cup C_{k}$, there exists a set $V^{\prime} \subseteq W$ with the following three properties:

(i) The subgraph of $(W, F)$ induced by $V^{\prime}$ is isomorphic to $(V, E)$ as a geometric graph,

(ii) $V^{\prime} \subseteq C_{i_{0}}$ for some $i_{0}$,

(iii) the convex hull of $V^{\prime}$ contains no point of $W \backslash V^{\prime}$. 
3. The above proof of Theorem 6 does not guarantee that $\operatorname{conv}\left(Q^{\prime}\right) \cap R=Q^{\prime}$. We do not know whether Theorem 6 with the extra condition $\operatorname{conv}\left(Q^{\prime}\right) \cap R=Q^{\prime}$ holds. In general we have the following question: Does there exist a planar point set $\bar{Q}$ for which an analogue of Theorem 6 holds but such an analogue does not hold if we further require that the corresponding set $\bar{Q}^{\prime}$ should satisfy $\operatorname{conv}\left(\bar{Q}^{\prime}\right) \cap R=\bar{Q}^{\prime}$ ?

4. The minimal size of the set $R$ in Theorem 3 is bounded by a primitive recursive function (by Shelah's proof of the Hales-Jewett Theorem, [Sh 88]). However, the best lower bound we have is only quadratic (in $|P|$ ). The quadratic lower bound holds even if we delete condition (iii) in Theorem 3.

5. If we delete condition (iii) in Theorem 3 then, for $k=2$ and for any set $P$ in general position, it is possible to find a set $R$ of size $\mathcal{O}\left(n^{2}\right)$ satisfying Theorem 3 (without (iii)), where $n=|P|$. On the other hand, for any positive integer $n$, there is a set $P$ of size $n$ in general position for which the size of any set $R$ satisfying Theorem 3 (without (iii)) is at least $\Omega\left(n^{2} / \log n\right.$ ).

Acknowledgment. We would like to thank the referee for his very careful work.

(This paper is to appear in Combinatorics, Probability and Computing (1994).)

\section{References}

[BF 87] I. Bárány and Z. Füredi, Empty simplices in Euclidean space, Canadian Math. Bull. 30 (1987), 436-445.

[ES 35] P. Erdős and G. Szekeres, A combinatorial problem in geometry, Compositio Math. 2 (1935), 463-470.

[GP 91] J.E. Goodman and R. Pollack, The complexity of point configurations, Discrete Appl. Math. 31 (1991), 167-180.

[GP 93] J.E. Goodman and R. Pollack, Allowable sequences and order types in discrete and computational geometry, in: J. Pach (ed.), New trends in discrete and computational geometry, Springer-Verlag (1993).

[GRS 80] R.L. Graham, B. Rothschild, and J. Spencer, Ramsey Theory, J. Wiley \& Sons, New York (1980).

[HJ 63] A.W. Hales and R.I. Jewett, Regularity of Positional Games, Trans. Am. Math. Soc. 106 (1963), 222-229.

[Ha 78] H. Harborth, Konvexe Fünfecke in ebenen Punktmengen, Elem. Math. 33 (1978), 116-118.

[Ho 83] J. D. Horton, Sets with no empty convex 7-gons, Canadian Math. Bull. 26 (1983), 482-484. 
[KL 85] B. Korte and L. Lovász, Posets, matroids, and greedoids, in: L. Lovász and A. Recski (eds.), Matroid theory, North-Holland (1985), 239-265.

[KLS 91] B. Korte, L. Lovász, and R. Schrader, Greedoids, Springer-Verlag, Berlin 1991.

[Lo 79] L. Lovász: Topological and algebraic methods in graph theory, in: A. Bondy and U.S.R. Murty (eds.), Graph Theory and Related Topics, Academic Press (1979), 1-14.

[MP 93] W. Moser and J. Pach, Recent developments in combinatorial geometry, in: J. Pach (ed.), New trends in discrete and computational geometry, Springer-Verlag (1993).

[NPT 81] J. Nešetřil, S. Poljak, and D. Turzík, Amalgamation of matroids and its applications, J. Comb. Th. B, 31 (1981), 9-22.

[NR 90] J. Nešetřil and V. Rödl (eds.), Mathematics of Ramsey Theory, Springer-Verlag (1990).

[NV 93] J. Nešetřil and P. Valtr, Order types containing approximately an affine transformation of the grid $k \times k$, preprint.

[Pa 91] J. Pach, Notes on Geometric Graph Theory, DIMACS Series in Discrete Mathematics and Theoretical Computer Science, Vol. 6 (1991), 273-285.

[Ra 30] F.P. Ramsey, On a problem of formal logic, Proc. Lond. Math. Soc., II. Ser. 30 (1930), 264-286.

[Sh 88] S. Shelah, Primitive recursive bounds for van der Waerden numbers, Journal AMS (1988), 683-697.

[Va 92] P. Valtr, Convex independent sets and 7 -holes in restricted planar point sets, Discrete Comput. Geom. 7 (1992), 135-152. 